\title{
A formula for creation and its applications to generate plots on computers
}

\author{
Doan Nguyen Hai
}

\section{The creation formula}

Among human intelligent activities, cration is always considered one of the superior forms. Creation, said briefly, is to produce somthing new in quality. To the question "Is Artificial Intelligence possible?", if we have some theory about machine creativity and several programs illustrating it. wo will have more confidence and evidence to answer 'Fes' or even 'No'.

1) First we consider the extend of creation. To us. creation is both original and universal.

(a) In common view, creation is often thought to be original. It is somthing which occurs only once. Nobody has ever done it before. or the creating agent does not know whether so. Creation comes to us sudienly, in sense of we can not be sure of it. Thus creation contains much interesting secret.

(b) But creation is universal. Indeed. if we agree that all events in this universe occur uniquely, then excry intelligent activity is creative. So it is a good idea to think of creativity as a fuzzy concept: all intelligent activities are creative, more or less.

Both the originality and the universality of creation prove the necessity to dral with creation in $\mathrm{AI}$. Creation is universal. so $\mathrm{AI}$ machines should be able to do that everyday activity. Creation is original, so AI would be successful only if it can do that high-quality task. 
2) The creation formula

$$
\text { Cration = Linowledge }+ \text { Logic }+ \text { Incidentalness. }
$$

Saying that creation las no formula is more acceptable than giving one to shape it. But we'd rather have a theory, a model. though not quite exact, than none.

We think that Creation is a compound of thrce substances which are kinowledge. Logic and Incidentalness. But let 's malyse each of them.

(a) The first component, knowledge, will get easy agrecment of most people. In general, to create, one need profound knowledge in the working domain. One of AI methodological sayings is 'Intelligenc'e needs knowledge'. Profound knowledge helps the creative agent discover the problem nature and the characteristic relations in it to produce new things.

(b) But knowledge is not enough to create. We nced a reasoning process to go from what we have had to what we have not, that is logic. Logic is the reasoning process, (iood logic implies good results.

(c) If knowledge and logic require true work and talent, incidentalness seems to be the incamation of unjustice. It exists in what we call good luck, what we call sudden. With the same takent and work, someone creates but the others don't. Or even the ereator is of less talent and does less work.

All the substances demonstrate the originality and the universality of creation. Incidentalness surely implies the originalify of creation but so do knowledge and good logic. Knowlerlge and togic control all intelligent activities but so does incidentalness because it is natural character of our unirerse and thus creation cannot be an exception.

\section{The plot generation system}

The Plot generation syste $m$ is a software which is able to generate plot of nord. play or film. Its creative principle is our creation formula. Its purpose is to faror the argument that machine can create, at least when riewed at the result, and to illustrate the creation formula. The requiment for the plots generated is to be 'human', that moans they must seen to be created by human, with logical, interesting, and some philosophical tharacteristics.

\section{a.) The basis model}

A plot consists of many erents. At the begining, there some initial events - they can be entered by the user, or can be generated at ranrlom, or can be selected (by user or at random) from a list of initial event sets arailable in the system.

Fx: This initial set consists of 3 erents:

$A$ is rich. B is pretty. Bunts to decciec $A$. 
The generation of a plot is carried on in two directions:

(1) The developing direction: In this direction, events are generated consecutively as logical consequences of each other.

Ex: From the above set, these events could be generated:

$A$ is rich. $B$ is pretty. $\Rightarrow A$ loots $B$.

$A$ loves $B . \Rightarrow A$ and $B$ marry.

$A$ and $B$ marry. $B$ wants to deccive $A . \Rightarrow B$ holds all $A$ 's property and then abandon $A$.

$B$ holds all $A$ 's property and then abadon $A . \Rightarrow A$ hates $B$.

A hates $B . \Rightarrow A$ kills $B$.

$A$ kills $B . \Rightarrow B$ dics. $A$ is arrested.

(2) The expanding direction: In this direction, events are expanded into rich details. In this way, the plot becomes more interesting.

Ex: Expand some events:

$A$ is rich. - $A$ is the boss of a big company

$B$ is pretty. $\rightarrow B$ is the Miss of the town.

$A$ kills B. - A poissons B.

$B$ dies. $\rightarrow B$ is taken to the hospital but it is uscless.

The materials used to produce the events are knowledge taken from the knowledge bases. In general, each category (social, sentimental. science-fiction, ...) has its own knowledge bases because it category has particular charactiristics on the content of the events. One can even divide a category into subcategories each of which has its own knowledge bases.

The knowledge bases contain knowledge in two kinds of rules: developing rules and expanding rules.

Ex: Some developing rules:

$A$ is rich. $B$ is pretty. $\Rightarrow$

A loves B. - A loves B. B loves A. - A loves B. B does not love A. - A loves $B$. B loves only $A$ 's property.

$A$ loves $B$. B loves $A . \Rightarrow$

$A$ and $B$ marry. $A$ and $B$ live happily. $-A$ and $B$ marry. $A$ and $B$ live unhappily.

$-B$ dies. $A$ is very unheppy.

$A$ loves $B . \Rightarrow$

$A$ and $B$ marry. $-B$ does not love $A$. $A$ is very unheppy. - $B$ marries $C . A$ hates $B$.

Thus a developing rule has form of:

Cause set $\Rightarrow$ Result set $1-$ Result set $2-\ldots$ 
Ex: Some expanding rules:

$A$ is rich - $A$ is the boss of a big company. - $A$ is a very rich man. - A's father is a millionaire.

A kills B. $\rightarrow$ A poisons B. - A shoots B. - A drouns B.

Thus an expanding rule has a form of:

An event $\rightarrow$ Set of detailled event: 1. - Set of detailled events 2. - . .

The basic work of the plot generation goes in the developing direction. From the current event set (at the begining, it is the initial set), the system looks for an applicable developing rule, then apply it to generate new events by taking at random a result set on the righ-hand side of the rule.

In general, a rule is applicable to the current event set if there is a subset of this set which can be matched with the left-hand side of the rule. (Matching is tried between the names of characters in the current event set and the variables in the rule. A variable occurring in the righ-hand side but not in the left-hand side will corresponding to a new character). Moreover, there are other criteria:

(1) It is not acceptable a repetition of a pevious application. The reason is to avoid generating the same events again.

(2) A rule is applicable only if the characters matched with its variables are still alive.

(3) If there are more than one applicable rules the one with the longest left-hand side is preferred.

(4) Still, if ther are more than one applicable rules, the choice is at random.

The generation of events will finish when there are no more developing rules to apply or when the number of events generated has exceeded a predefined limit.

The last stage is to expand the events into detailled events using the expanding rules. An event is expanded by taking incidentally a set of detailled events on the righ-hand side of the matched rule. If there are no matched rules, the event is matched to itself. Finally, the detailled events are printed as the produced plot.

\section{Principal characters}

With the basic model, we will have unpredicted various plots, in each of which there could be some characters emerging as principal or there could be no principal characters at all. Now we propose is solution to set up principal characters before generation."

It can be thought simply that a character is principal when the proportion of events in which that character occurs to the total events (called the occur proportion) is greater than some predefined limit. In our experimental system, we have chosen 
that limit $=60 \%$. Similarly, we can establish the occur proportion of important subordinate characters from $10 \%$ to $60 \%$.

The generation process now has some little additions:

(1) The principal character is established before the generation. And when lis occur proportion in the current set is $<60 \%$. the rule selected should generate new events such that this proportion get $s \geq 60 \%$. Otherwise all goes normally. (Thus if the principal character has died byt his occur proportion is still $\geq 60 \%$, more events could be generated as the tail of the plot. But, as this proportion decreases to $60 \%$ the process terminates.)

(2) Similar manipulation for important subordinate characters.

(3) With other characters the system sould control their occur proportions not exceeding the principal character's occur proportion.

\section{b. The structural model}

The basic model produces various plots not obering any user's intention. For example, the system could give out a plot in which good people suffer much while evil-doers enjoy all pleasures. So now we propose the structural model. which is developed from the basic model and is able to gencrate plots following a structure.

For instance. we want to have plots with the philosophy 'Good defats Evil'. Such a philosophy can be expressed in the structures in the following structure rule:

Good defeats Evil $\Rightarrow$

$A$ is good and B is cril. B allacks $A$. A punished $B$ successfully. -

$A$ is good and $B$ is coil. $B$ attacks $A .1$ punished $B$ unsuccessfully. $B$ attacks A. B punished A successfully. -

$A$ is.good and $B$ is evil. $B$ attacks $A$. A forgives $B$. B feels remorse.

This structure rule has thliree structures on the right-hand side. In general, our system can contain many structure rules for vatious philosophies (such as 'Evil defeats (iood', 'Love overcómes all hatreds', 'Might is right', etc.) and each structure rule can have many structures. To produce a plot wich follows a philosophy, the system chooses incidentally a structure in the corresponcling structure rule.

The erents in a structure are called the frame erents. For instance, the second structure in the above example consists of 5 frame events. We will use this structure to illustrate the generation process.

Suppose the initial set is:

$A$ is rich. $B$ is poor.

The generation process begins with the expansion of the first frame event. Then the developments and expansions are carried on as in the basic model. Next we continue all such things for the next frame cvent, then for the next gframe event, 
$\ldots$, till the end of the structure. If the structure has $n$ frame events the gencration process is a for-loop:

for $i=1$ to $n$ do

begin

$\{$ Stage $i\}$

Expand the $i^{t} h$ frame event;

Develop and expand the events as in the basic model; end;

The expansion of a frame event is due to the frame expanding rules.

Ex: Some frame expanding rules:

$\left\{\begin{array}{l}A \text { is good and } B \text { is evel. } \\ A \text { is rich. } B \text { is poor. }\end{array}\right.$

$A$ is very kind and honest. $B$ is ambitious and cruel. -

A often helps people. $B$ is deceitful and ambitious.-

A often helps B but B is very ungrateful.

$A$ is good and B is evil. $\Rightarrow$

$A$ is very kind and honest. $B$ is wicked and ambitious.-

A always does good thing. $B$ is selfish, ambitious and hypocritical.-

$\left\{\begin{array}{l}\mathrm{B} \text { attacks } \mathrm{A} . \\ \mathrm{A} \text { is rich. }\end{array} \rightarrow\right.$

$B$ robs A's property.-- $B$ deceives $A$ to scize A's property.

$B$ attacks $A$. $\Rightarrow$

B harms A. - B insults A.- C is A's friend. B harms C.-

$B$ wants to do evil things but $A$ hinders $B$. B harms $A$.

And suppose we have the following developing and expanding rules:

$B$ deceives $A$ to seize $A$ s property. $\rightarrow$

$B$ cheats $A$ in playing cards and wins all $A$ 's property.-

$B$ cooperates with $A$ and deceives $A$ to hold all $A$ 's capital.

$B$ deceives $A$ to seize $A$ 's property. $\Rightarrow$

$A$ becomes poor. $B$ becomes rich. $-A$ suicides. $B$ becomes rich. $-A$ becomes poor. $B$ becomes rich. A hates $B$.

$A$ becomes poor. $B$ becomes rich. $\Rightarrow$

$C$ is $A$ 's lover. $C$ abadons $A$ to go with $B$.-

$A$ works for $B$. $B$ insults $A$.

Then we could have the first events of the plot as follow: 
$A$ is rich. $B$ is poor. A often helps people. $B$ is deceilful and ambitious. B cheats $A$ in playing cards and wins all $A$ is property. I becomes poor. B becomes rich. $C$ is $A$ 's lover. C abandons A to go with $B$.

We have said that if the structure has $n$ frame events the process will go through $n$ stages. One important thing is the attachment betwwen the events in the previous stages and the succeding satges. For instance, we'd rather have a frame structure rule like:

$$
\left\{\begin{array}{l}
A \text { punishes } B \text { unsuccessfully. } \\
C \text { is } A \text { 's lover. C abandons } A \text { to go with } B .
\end{array} \Rightarrow\right.
$$

A fails to kill $B$ but kills $C$ insterd.-

$A$ just could kill $B$ if $C$ did not hinder 1.

The attachment between stages sometimes requires a backtracking from a stage to its previous stages. For instance, if the stage ' $B$ attachs $A$ ' has led to the event 'A dies' the process should backtrack to delete this event and to produce another one in order to be able to go on to the next stage ("A punishrs $B$ unsuccessfully). However. there is also another way to avoid backtracking, by including the knowledge base rules like:

$$
\left\{\begin{array}{l}
A \text { A punishes } B \text { unsuccessfully. } \\
A \text { dies. }
\end{array} \Rightarrow\right.
$$

$A$ is not really dead. A is saved by $C$. A punishes $B$ unsuccessfully.-

$A$ is incarnated into $C$. A punishes $B$ unsuccessfully.

Other applications of the structural model

The structural model is not only applicalsle to generate plots with philosophis cal intentions but in gencral, it can be used to produce plots that should have a struct ure.

A good example is the detective plots. The basic model seems unsuitable for this kind which requires a good arrangement of events. But the structural model is promising:

\section{Detective plots $\Rightarrow$}

$A$ is killed. B,C.D are suspect. B, ('are proved innocent. $D$ is proved the murderer.-

$A$ is killed. B,C,D are suspect. B,C,D are prored the murderers.

Another application of the structural model is we can build structures having branching points. At the points, the plot may continue in one of many possibilities the generation process may pause, list these possibilities and wait the user's choice. 
Applications of the plot gr meration system

The plot generation system can be used

1) To prove the possibility that machine can do creative acts.

2) to gencrate plots og novel. pay or films for human writers.

3) to generate animated cartoons when combined with a drawing system.

The basic and structural models can be used to generate pictures and music.

The creation formula can be applied for other syustems doing creation and can be used as a theoretical molel for creative activities.

\section{For conclusion: the predestination motivation}

Regarding human creation. there are many options about the motiation. Some think that it is the social motiation; to live and to terelop, humanity must to creative acts. Some thinh that libido causes all. We do not consider what the motiration is here but we would like to present the idea that the motivation. whateres it be, is the one who has put the + and = signs into the creation equation.

Regarding machines. particulasly the plot generation system, we call their motivation of creation a predestimation motiration: machines do cractive acts because I have programmed them to do so. As a matter of fact. man man does not always why hi lives; is there any programmer who has programmed us to live in his program. there is some looseness called incidentalness:"

\section{Abstract}

In this paper we propose "formula for creation and present the design of a plot grencration system as one application of this formula. Such a system has been implemented in our uniersily in 199.3.

Department of Computer Scinece, Polytechnical Enirersity of HoChiMlinh City 268 Ly Thuong Kiet Str. HoChiMinh City: 